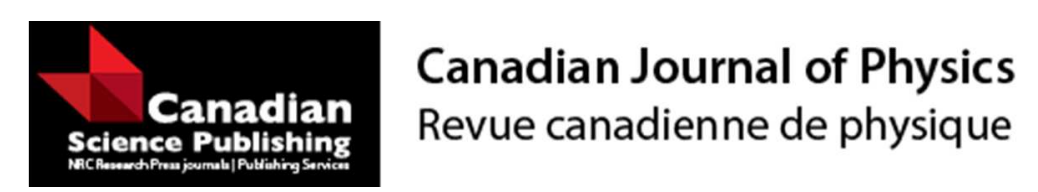

\title{
Deformation and rupture of vesicles confined in narrow channels
}

\begin{tabular}{|r|l|}
\hline Journal: & Canadian Journal of Physics \\
\hline Manuscript ID & cjp-2016-0796.R1 \\
\hline Manuscript Type: & Article \\
\hline Date Submitted by the Author: & 08-Feb-2017 \\
\hline Complete List of Authors: & $\begin{array}{l}\text { Harman, Alison; University of Ottawa, Physics } \\
\text { Bertrand, Martine; University of Ottawa, Physics } \\
\text { Joos, Bela; University of Ottawa, Physics }\end{array}$ \\
\hline Keyword: & vesicle, molecular dynamics, microfluidics, lipid bilayers, Iysis \\
\hline Please Select from this Special & \\
Issues list if applicable: & \\
\hline
\end{tabular}

\section{SCHOLARONE ${ }^{\text {Tw }}$ \\ Manuscripts}




\title{
Deformation and rupture of vesicles confined in narrow channels Déformation et rupture des vésicules dans des canaux étroits
}

\author{
Alison Harman, Martine Bertrand, Béla Joós \\ Ottawa-Carleton Institute for Physics \\ University of Ottawa Campus \\ Ottawa, Ontario, Canada, K1N 6N5
}

(Dated: February 8, 2017)

\begin{abstract}
Using coarse-grained molecular dynamics simulations, we investigate the rheological properties of lipid bilayer vesicles as they travel in tight capillaries such as those found in the vasculature and micro-fluidic devices. By varying the channel size, we study the build-up of tension as the flow increases with the aim of predicting the location of lysis and the mechanisms of rupture. Highly confined, fully inflated vesicles show the greatest stress and rupture near their front tip. We also simulate vesicles with reduced volume $\nu=0.6$, the same reduced volume as red blood cells, to show how stress builds up in those objects in various conditions.

Utilisant des simulations de dynamique moléculaire à gros grains, nous étudions les propriétés rhéologiques de vésicules avec bicouches lipidiques, se déplaçant dans des capillaires étroits comme ceux qui se trouvent dans la vasculature et les dispositifs microfluidiques. En variant la taille des canaux, nous avons étudié l'accroissement de la tension avec l'augmentation du flot pour déterminer la location du point de rupture et ses mécanismes. Les vésicules fortement confinées et complètement gonflées exhibent la plus grande tension et une rupture près de leur extrémité avant. Nous avons aussi simulé des vésicules avec le volume réduit $\nu=0.6$, égal à celui des globules rouges de sang, pour illustrer comment la tension se développe dans ces objets dans des conditions différentes.
\end{abstract}




\section{INTRODUCTION}

Vesicles are closed fluid phospholipid membranes suspended in a viscous solution. As such, they can experience a variety of non-linear behaviour under different flow rates, enclosed volumes, and membrane composition. Artificial vesicles (also known as liposomes) are used as drug carriers [1,3] and in the study of biomembrane mechanics [4]. For the properties of vesicles suspended in microfluidic channels, multiple experimental [5, 6] and theoretical/computational studies [7-14] have been conducted on this topic. However, few address the issue of rupture due to stresses induced by hydrodynamic interactions with the surrounding fluid [15]. Consider, for example, small unilamellar vesicles (SUVs) that are to be prepared and loaded with a potent molecular agent to be delivered to a specific site. It would be highly useful to know if, when, and where rupture and content leakage occur. In this report, we shed light on this phenomenon via coarse-grained molecular dynamics (CGMD) simulations, using the simplest model that contains the main feature of lipid bilayers, namely that they are fluid within the plane.

Few experimental studies have been conducted on the migration and shape deformation of single vesicles [5, 6] or red blood cells (RBCs) [16, 17] in bounded Poiseuille flow. Vitkova et al. [5] and Coupier et al. [6] measured shape deformation as a function of vesicle velocity, reduced volume, and vesicle size relative to channel size. These studies consider stable shapes and do not examine rupture nor tension in the bilayer.

There are numerous theoretical and computational studies that focus on the deformation of vesicles in bounded flows. It is possible to find analytical solutions to the simplest situations only, such as when vesicle deformations are small [13]. Anything else requires the use of numerical techniques or simulations. The Boundary Integral Method (BIM) is a popular approach to numerically solve for a vesicle immersed in a solvent: constitutive equations for the elastic properties of the membrane are coupled to Stokes equations for an incompressible fluid at low Reynolds number. The response of the lipid bilayer membranes is modelled according to Helfrich's free-energy [18]. The BIM has been applied to vesicles transported by flow in channels $[8,10,19,20]$. Others have opted for particle based simulations, where the membrane is a mesh and the fluid is made of point particles [7, 21]. They work under the common assumption that lipid membranes offer a large resistance to stretching but little to bending with a conserved volume and local surface area. We simulate vesicles via CGMD 
where both the lipids and the solvent are explicitly modelled. No constraints on area expansion of the membrane or internal volume are imposed. As such, it is possible to model both unstable vesicles and subsequent rupture due to hydrodynamic stresses which, to the best of our knowledge, has not been done yet.

Vesicle lysis has been studied in various contexts such as osmotic pressure [22], micropipette aspiration [23 25], and acoustic streaming [26]. However our study of vesicle rupture in the context of flow driven transport down a narrow channel is novel. Using CGMD, we simulate the migration of capillary confined SUVs under moderate to high flow strengths such that the dominating factor that determines shape deformation should be the hydrodynamics rather than the specific details of our model. We vary both the size of the channel with respect to a SUV of constant size and the applied pressure gradient along the length of the channel. Our simulations show rheological properties of fully-inflated vesicles flowing down narrow channels in agreement with previous experimental studies. In addition, we study the resulting stress and rupture point along the membrane. Vesicles with the reduced volume $\nu=0.6$ were also studied to compare their biomechanics with red blood cells (RBCs) as RBCs have the same reduced volume and are known to share some similar behaviour under flow.

\section{METHODS}

\section{A. Simulations}

We conducted CGMD simulations following the model used in recent studies by Bertrand et al. [27] and Barlow et al [28], inspired by Goetz and Lipowsky's coarse-grained simulations of lipid bilayers [29]. The lipids, which compose our vesicles, consist of three particles, or beads, with one for the hydrophilic head and two for the hydrophobic tail. The solvent is also represented by beads and interacts via the Lennard-Jones (LJ) force. The heads interact with the solvent via the LJ potential while the tails do so via a softer and purely repulsive potential. The units of length and energy are respectively the $\sigma$ and $\epsilon$ of the LJ potential. Each particle in the system has the same mass $m$. Hence the unit of time is $\tau=\sqrt{m \sigma^{2} / \epsilon}$.

Bertrand et al. have characterized a bilayer membrane made of our model lipids in a LJ solvent of density $\rho=0.8 \sigma^{-3}$ and a shear viscosity $\eta=1.98 \pm 0.16 \sigma^{-2} \sqrt{m \epsilon}$. At 
a temperature of $k_{b} T=1.0 \mathrm{\epsilon}$, it was found that the unstressed area per lipid head was $a_{0} \cong 1.9 \sigma^{2}$, the equilibrium bilayer thickness $l \cong 4.8 \sigma$, and the area compression modulus $K_{A}=8.84 \pm 0.76 \epsilon / \sigma^{2}$. The bending modulus was approximated to be $\kappa \cong K_{A} l^{2} / 24=$ $8.48 \pm 0.72 \epsilon$ [27]. Our lipid bilayer is therefore "soft" as the bending modulus is the same order of magnitude as the thermal energy.

A vesicle composed of 3000 lipids was found, at equilibrium, to have an outer radius of $R_{0}=16.5 \sigma$ with 1841 lipids and an inner radius of $R_{i}=11.5 \sigma$ with 1159 lipids. The outer and inner leaflets (referred to with the + and - symbols respectively) have different equilibrium areas per lipid head with $a_{0,+}=1.86 \sigma^{2}$ and $a_{0,-}=1.68 \sigma^{2} \cdot a_{0,+}$ is close to the unstressed area per lipid head of a bilayer., $a_{0} \cong 1.9 \sigma^{2}$ The inner layer is more compressed than the outer layer, but this is characteristic of self-assembled small unilamellar vesicles [30]. We define an average equilibrium area per lipid for the vesicle $a_{a v g, 0}=\left(a_{0,+}+a_{0,-}\right) / 2=$ $1.77 \sigma^{2}$.

Our main priority in setting up our coarse-grained model was to include the in-plane fluidity of lipid bilayers, a unique mechanical property of these membranes. The particles in our system do not correspond to specific molecules, and as such establishing a correspondence to SI units requires some interpretation. Our lipids are considered to be crude approximations to two-tailed lipids: each tail bead can be defined as two strands of five $\mathrm{CH}_{2}$ groups which gives a length scale of $\sigma \approx 0.6 \mathrm{~nm}$ and a mass scale of $N_{A} m \approx 140 \mathrm{~g} / \mathrm{mol}$. And assuming a constant temperature $T=310 \mathrm{~K}$, this results in the unit of energy $\epsilon=4.27 \times 10^{-23} \mathrm{~J}$.

With these estimates, the thickness of our bilayer membrane is approximately $2.88 \mathrm{~nm}$. This is less than the typical thickness of around $5 \mathrm{~nm}$, but our membrane properties are within the lower, but acceptable range, for lipid bilayers. We find the bending modulus to be $\kappa \approx 3.6 \times 10^{-21} J$ as compared to typical values of around $10^{-20} J$ [31]. As we work in strong flow, bending forces will be weak and have little impact on the kinetics.

We apply a constant force to all particles in the simulation; this is the equivalent to a constant pressure gradient along the capillary. Poiseuille flow is recreated in the absence of the vesicle. In order to avoid sudden stresses to the vesicles, we submitted the vesicle to a steadily increasing force per particle. The loading rate was chosen to allow the vesicle time to equilibrate between two successive increments of the force. It was kept constant between different simulations.

The flow around the vesicle is laminar. The largest Reynold's numbers occur in the case https://mc06.manusc/jiptcentral.com/cjp-pubs 
of the least confined vesicle at the highest velocities. Reynold's number, $R e$, is defined as $R e=v \rho L / \eta$ where $L$ is a characteristic dimension and $v$ is the velocity of the vesicle. For the largest channel, $L$ is the pipe diameter which in Lennard-Jones units is $27.3 \sigma$. Using the values of $\eta=1.98 \pm 0.16 \sigma^{-2} \sqrt{m \epsilon}, \rho=0.8 \sigma^{-3}$ [27] and our highest velocity of $v \approx 1.0 \sqrt{\epsilon / m}$, we find our largest Reynold's number to be $\mathrm{Re} \approx 7$.

\section{B. Analysis}

To look at the effect that the imposed fluid flow has on the vesicle, we triangulate the positions of the outer and inner lipids heads using the Crust surface reconstruction algorithm [32. From the known positions of the lipid heads, the Crust algorithm constructs a surface of triangles whose vertices are the lipid head positions. This algorithm assumes a continuous surface. From these triangles, one is able to calculate an area per lipid head, $a$. Our vesicle is curved and in order to extract an area expansion for the bilayer, we average the area expansion and compression of the outer and inner heads respectively in order to find the average area per lipid in a section of membrane: $a_{\text {avg }}$ equals $\left(a_{+}+a_{-}\right) / 2$ where $a_{+/-}$is the average area per lipid for the outer $(+)$ or inner $(-)$ monolayers respectively. The strain in the bilayer is measured by the fractional area expansion:

$$
\alpha_{v e s}=\frac{a_{a v g}-a_{a v g, 0}}{a_{a v g, 0}}
$$

where $a_{a v g, 0}=\left(a_{0,+}+a_{0,-}\right) / 2=1.77 \sigma^{2}$, is the average area per lipid at rest to be compared with the flat membrane area $a_{0} \sim 1.9 \sigma^{2}$ At moderate forces, the strain, $\alpha_{v e s}$, is linearly proportional to the stress or tension $\gamma$ through the area compressibility constant $K_{A}$, i.e. $\gamma=K_{A} \alpha_{\text {ves }}$. At a critical area expansion (or, equivalently, a critical tension) the bilayer is prone to form pores.

In order to have a dimensionless format in which to express the applied pressure gradient and the resulting velocities, we look at the defining characteristics of our system. We have a known bending modulus, $\kappa$, of our bilayer, an initial radius of our vesicle $R_{0}$, the viscosity of the external fluid $\eta$, and the area compressibility modulus $K_{A}$ [27]. The values of these constants can be found in the previous section which describes the simulation model. The units are based on the Lennard-Jones (LJ) force between like particles, the mass of the particles and the constant temperature of the system. From these constants one can look at 
the time-scales which define the system. There is a time-scale associated with bending [8]:

$$
\tau_{\text {bend }}=\frac{R_{0}^{3} \eta}{\kappa}
$$

a time-scale associated with stretching

$$
\tau_{\text {stretch }}=\frac{\eta R_{0}}{K_{A}},
$$

and a time-scale associated with the shear rate of the flow $\dot{\gamma}$

$$
\tau_{\text {flow }}=\frac{1}{\dot{\gamma}}=\frac{\Delta y}{\Delta U}=\left(\frac{\Delta P}{\Delta L} \frac{R_{0}}{2 \eta}\right)^{-1}
$$

within Poiseuille's approximation. $\Delta U / \Delta y$ is the rate of change of the velocity $U$ of the fluid in the direction $y$ perpendicular to the flow.

Substituting our values for $R_{0}, K_{A}, \kappa$, and $\eta$, we find that $\tau_{\text {bend }} \sim 2600 \tau$ and $\tau_{\text {stretch }} \sim 5 \tau$. This means that bending forces are very weak and vesicle shape is controlled by stretching. With weak bending forces the vesicle takes a long time to relax after a pertubation. The ratio of $\tau_{\text {bend }}$ to $\tau_{\text {stretch }}$ is for our model membrane,

$$
\frac{\tau_{\text {bend }}}{\tau_{\text {stretch }}}=\frac{R_{0}^{2} K_{A}}{\kappa} \approx 1000,
$$

in which the viscosity of the external fluid cancels. For the last time constant, if we substitute a typical value for the pressure gradient, $\Delta P / \Delta L \sim 0.01 \epsilon / \sigma^{4}$, we find that $\tau_{\text {flow }} \sim 30 \tau$. Our observations are taken every $5000 \tau$ and therefore our vesicles are steady-state objects at the time of observation for moderate forces. At high forces, vesicles become unstable such that rapid stretching of the bilayer occurs.

From these characteristic times, one can define dimensionless capillary numbers $C a_{b}$ and $C a_{s}$, which are proportional to the shear rate (a controlled variable) $\dot{\gamma}$. They measure the relative strength of the fluid friction force relative to the strength of the vesicle with respect to bending $\left(C a_{b}\right)$ or stretching $\left(C a_{s}\right)$. We have:

$$
\begin{gathered}
C a_{b}=\frac{\text { friction force }}{\text { bending force }}=\frac{\eta \frac{\Delta U}{\Delta y} \pi R_{0}^{2}}{\kappa \frac{\pi}{R_{0}}}=\left(\frac{\Delta P}{\Delta L}\right) \frac{R_{0}^{4}}{2 \kappa} \\
C a_{s}=\frac{\text { friction force }}{\text { stretching force }}=\frac{\eta \frac{\Delta U}{\Delta y} \pi R_{0}^{2}}{K_{A} \pi R_{0}}=\left(\frac{\Delta P}{\Delta L}\right) \frac{R_{0}^{2}}{2 K_{A}}
\end{gathered}
$$

The last term has been obtained within Poiseuille's approximation which yields $\Delta U / \Delta y=$ $(\Delta P / \Delta L) R_{0} /(2 \eta)$. It is to be noted that:

$$
C a_{b}=\frac{\tau_{\text {bend }}}{\tau_{\text {flow }}} ; \quad C a_{s}=\frac{\tau_{\text {stretch }}}{\tau_{\text {flow }}} ; \quad \text { and } \quad \frac{C a_{b}}{C a_{s}}=\frac{\tau_{\text {bend }}}{\tau_{\text {stretch }}}=\frac{R_{0}^{2} K_{A}}{\kappa} \approx 1000
$$


from Eq. 2.5.

These capillary numbers are independent of the viscosity of the fluid within the laminar flow approximation. The capillary numbers defined in equations 2.6 and 2.7 have been employed in several theoretical and experimental works used to describe vesicle shape deformations [10], [6], [13]. Capillary numbers give an indication of the effect of bending (or stretching) of the membrane as compared to the shear stresses imparted to the membrane. For low capillary numbers the two factors are comparable, and at high capillary numbers shear stresses dominate and determine the vesicle shape.

We will use the capillary number $C a_{b}$ in Eq. 2.6 for the analysis of our results since $C a_{s}$ and $C a_{b}$ are related by constant $\left(C a_{s} \approx C a_{b} / 1000\right)$. From here on $C a$ means $C a_{b}$ in Eq. 2.6.

\section{RESULTS}

We study two main families of vesicles with different types of reduced volumes $\nu$, the fully inflated vesicle with $\nu=1.0$ and a partially inflated vesicle with $\nu=0.6$. The reduced volume $\nu$ is defined as the ratio of the vesicle volume $V$ over the volume of the sphere with the same area $A$ as the vesicle:

$$
\nu=\frac{V}{\frac{4}{3} \pi(A /(4 \pi))^{3 / 2}}
$$

\section{A. Deformation of fully inflated vesicles (reduced volume $\nu=1.0$ )}

Before any force is applied, this $\nu=1.0$ vesicle is spherical except for thermal undulations. The vesicles are constructed to attain a state where the internal and external fluid pressures are equal. The pressure difference has two contributions, potential and kinetic. The latter, driven by temperature, leads to significant undulations that persist up to lysis tension [28]. When subjected to forces in the flow, although $\nu=1.0$, the simulated vesicle can deform for three reasons, the flattening of undulations, elastic stretch, and even some compression of the inner fluid. It is commonly thought that bilayers are inextensible, but in fact they can be stretched up to $4 \%$ as found by Olbrich et al. [33. At low tension regimes, it is mostly the flattening of thermal undulations but at high tension regimes area-stretch is the dominant effect as shown by Rawicz et al. [31]. In the experiments of Vitkova et al. [5] that we will discuss, the deformations are attributed to the first two reasons mentioned above, 
flattening of undulations and elastic stretching, and the outflow of water. In short, our value of $\nu=1.0$ is only meant to reflect the starting reduced volume of the vesicle.

1. Eccentricity and relative velocity

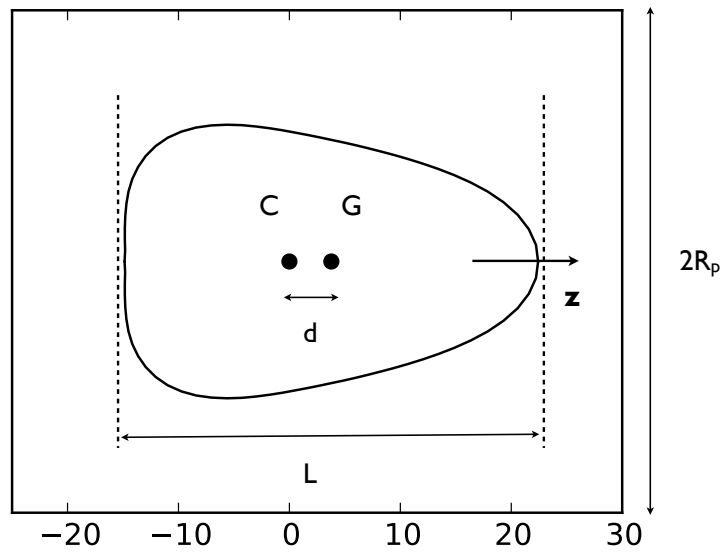

FIG. 1. A bullet-shaped vesicle for $\lambda=R_{0} / R_{p}=0.6$. The center of mass of the vesicle is labelled by $\mathrm{C}$ and the geometric center is labelled as G. Eccentricity is defined as $e=2 d / L$.

First, we measure the deformation of the fully-inflated vesicles $(\nu=1.0)$ using the metric of eccentricity. Eccentricity is defined as (see Figure 1) :

$$
e=\frac{2 d}{L}
$$

where $d$ is the distance between the center of mass and geometric center of a vesicle with length $L$. This calculation is done for the same confinements $\lambda$ as were used by Vitkova et al. [5] in order to compare our results with their experimental results:

$$
\lambda=\frac{R_{0}}{R_{p}}
$$

where $R_{0}$ is the vesicle radius and $R_{p}$ the pore radius. The confinement values used are $\lambda=$ $1.0,0.8$, and 0.6 .

In order to test the validity of our model, we modelled the experiment of Vitkova et al. [5]. We calculated the eccentricity of the vesicle and plot it as a function of its velocity (see Fig. 2). The results compare well with Vitkova et al.. In both cases the vesicle asymptotically 
approaches the same maximal eccentricity of about 0.16 with similar curves. Vesicles which are less confined can tolerate higher velocities and experience a more gradual increase in eccentricity. The shape of the vesicle as seen in Figure 1 is commonly known as a "bulletshaped" vesicle. At low velocities, the vesicle shapes are more ellipsoidal and have lower eccentricities than the shape represented in Figure 1 .

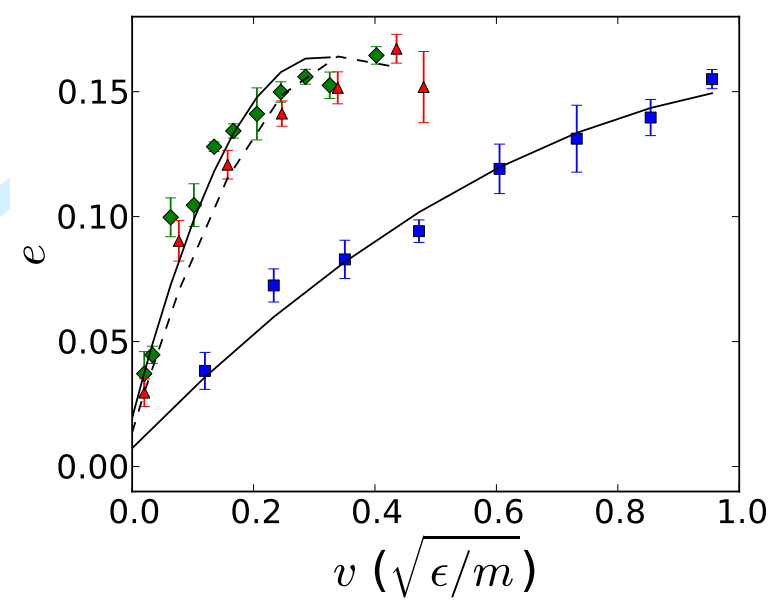

FIG. 2. Eccentricity, $e$, of a fully inflated vesicle versus velocity, $v$, for various confinements, $\lambda$, (where $v$ is the velocity of the vesicle): $\bullet=0.71, \boldsymbol{\Delta}=0.67$, and $\mathbf{\square}=0.43$. Lines are drawn through the points to guide the eye. This graph corresponds well to Ref. [5].

In addition one can analyze the ratio of the vesicle velocity versus the average fluid velocity $v_{v e s} / v_{\text {avg }}$ as a function of the pressure drop along the channel (see Fig. 3). Capillary number is proportional to pressure drop as defined in equation 2.6. Tomaiuolo et al. made this analysis RBCs in experiment [17]. Similar results are expected for vesicles and RBCs as their fluid dynamics along a channel are similar. Our relative velocity reaches a constant value depending on confinement. The most confined vesicles are moving closer to the average fluid velocity, i.e. $v_{v e s} / v_{\text {avg }}$ has a value close to one. For a lesser confined vesicle $v_{v e s} / v_{\text {avg }}$ has a value closer to two as the fastest fluid is at the center of the pipe and the lesser confined vesicle occupies a smaller cross section. In Poiseuille flow the maximum fluid velocity at the center of the channel is twice the average velocity of the fluid therefore the values of $v_{v e s} / v_{\text {avg }}$ range between one and two. The plateau values of around 1.6 and 1.2 are similar to what Tomaiuolo et al. find experimentally for similar confinement ratios [17]. From Fig. 2 and 
Fig. 3, our vesicles behave in similar ways to vesicles and RBCs under flow.

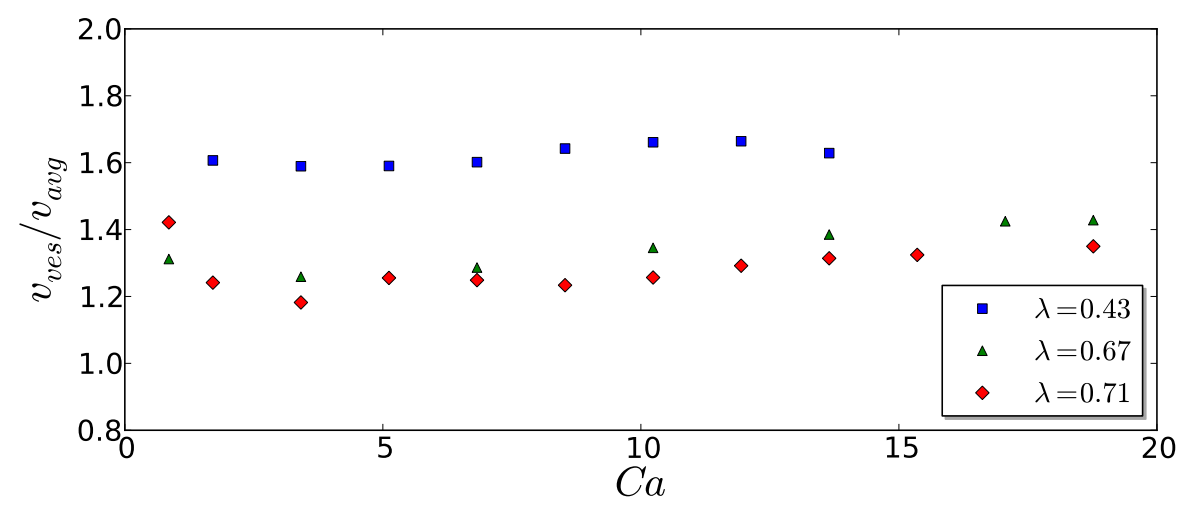

FIG. 3. Ratio of vesicle to average fluid velocity, $v_{v e s} / v_{a v g}$, as a function of capillary number, Ca, for various confinements, $\lambda: \bullet=0.71, \boldsymbol{\Delta}=0.67$, and $\mathbf{\square}=0.43$.

\section{Area expansion}

We divided the vesicle into sections along its length and calculated the strain $\alpha_{\text {ves }}$ (defined in Eq.2.1 for each section. We plot in Figure 4 this $\alpha_{\text {ves }}$ for several flow strengths measured by the capillary number $C a$. The dot with error bar indicates the location of rupture if $C a$ were increased further. This rupture occurs at the peak in the area per lipid for two values of the confinement $\lambda, 1.0$ and 0.8 . For low confinement $\lambda=0.6$, no rupture was observed.

The area expansion profiles show maximums where the lipids are most expanded and, for values of $\alpha_{\text {ves }}$ greater than zero, the bilayer is stretched above the equilibrium value of the $a_{a v g, 0}$ of the vesicle at rest. Area expansion depends both on confinement and capillary number $\mathrm{Ca}$. As the capillary number increases, there is an overall increase in the area expansion. For the same capillary number, the area expansion is much greater with the larger confinements. In addition there is a more pronounced peak in stress for the more confined vesicle around $z / L \approx 0.8$. For the smallest $\lambda$, of 0.6 , the stress profile of the vesicle is more flattened and of less magnitude compared to higher $\lambda \mathrm{s}$. Not surprisingly this vesicle does not rupture like the more confined vesicles. Instead there is a low or negative tension at the rear of the vesicle and it is from this area that tethers form. This is similar to what happens with membrane-pulling experiments. 


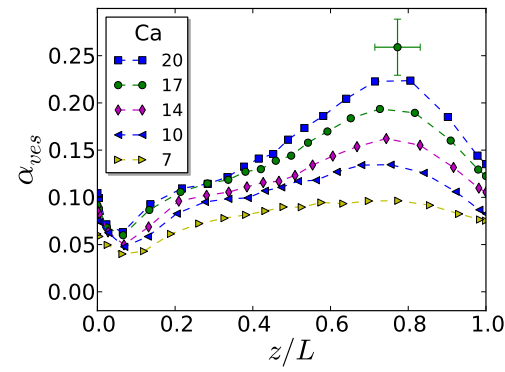

(a) $\lambda=1.0$

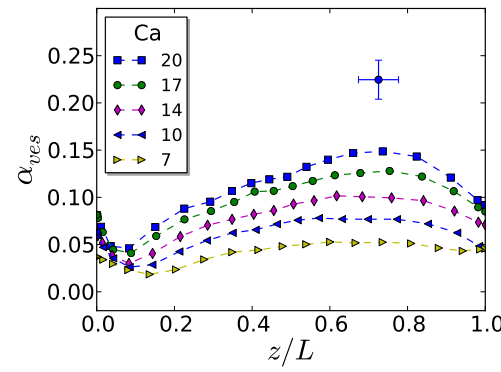

(b) $\lambda=0.8$

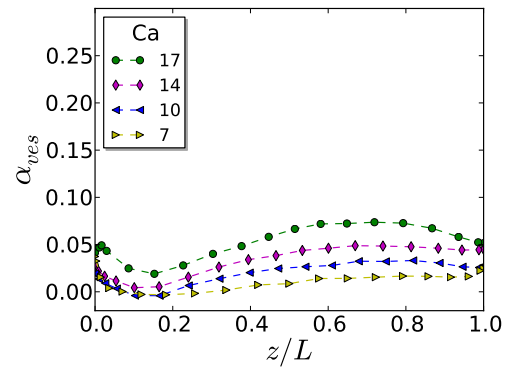

(c) $\lambda=0.6$

FIG. 4. The area expansion along the membrane as a function of the length of a fully inflated vesicle for different flow strengths. The $z$ coordinate along the length of the vesicle has been normalized between zero and one to account for different lengths of vesicle. Area expansion depends on confinement and capillary number. The most expanded area is where rupture will occur. The dot with error bars indicates the location of rupture and the area expansion of the lipids and its error. For the least confined vesicle, the area expansion of the lipids is not great enough to induce rupture.

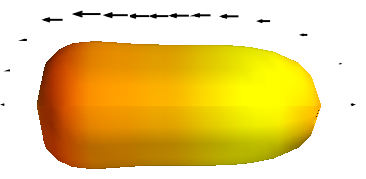

(a) $\lambda=1.0$

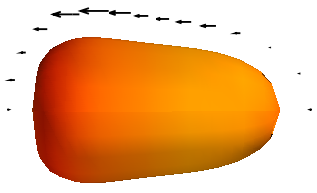

(b) $\lambda=0.8$

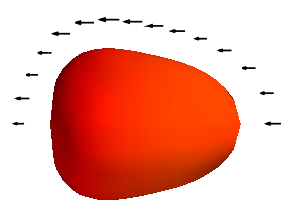

(c) $\lambda=0.6$

FIG. 5. Area expansion profiles of fully inflated vesicles for $C a=15$. The lighter (yellow) color indicates the point of highest stress and the darker (red) color indicates low tension. More confined vesicles exhibit greater local area expansions and are likely to rupture.

As mentioned above the area expansion of the lipids just before rupture is also shown in Figure 4 (a) and (b) at the dots with error bars. The area expansions of the lipids just before rupture for $\lambda=0.8$ and 1.0 are equal within error. To narrow the location of rupture, we visually review the location of rupture. The triangulation of the lipid heads provides the area expansion of the lipids. The peak locates the point of rupture even though the pore 
expands quickly once formed.

The area expansion profile was mapped onto the 3D re-creation of the average vesicle shape for capillary number $C a=15$ for three different confinement values (see Fig. 5). In addition the velocity of the fluid in the vesicle's frame of reference is shown. The flow around the vesicle is laminar. One can see for this pictorial representation that the most confined vesicle is the most stretched and consequently the longest vesicle. The lightest yellow color represents the largest local area expansion and the place which the vesicle will rupture first. The back of the vesicles are at very low tension.

\section{Rupture mechanisms}

Following the above observations made in Section III A 2, it is no surprise to see that fully inflated vesicles rupture near the front as seen in Fig 5. Fig. 6 (a) shows for a highly inflated vesicle $\lambda=1.0$ that rupture occurs with the nucleation of a pore from the outside. The outer leaflet expands enough such that the external fluid can infiltrate the bilayer and then the inner lipids move to avoid the fluid. As the simulation time progresses, the pore becomes larger and the vesicle loses all or most of its contents. Such very confined vesicles $(\lambda \gtrsim 0.9)$ can reassemble with large (greater than $50 \%)$ fluid loss.

For less confined vesicles, the rupture mechanism is quite different. Tether formation is observed (see Fig. 6(b)). The tether is usually a single tail micellar structure rather than a bilayer like the rest of the vesicle. The area expansion of the lipids is minimal at the rear of the vesicles and that means they could expand readily in response to forces. As the fluid is flowing past the sides of the membrane, once tether formation starts to occur, it continues until it breaks from the main body of the vesicle.

It was necessary to slowly increase the pressure gradient applied in order to minimize sudden stresses to the vesicle, as highly confined vesicles are likely to experience leakage if the loading rate is too high. This is true for both fully-inflated and vesicles with $\nu=0.6$ although vesicles with $\nu=0.6$ are more likely to experience leakage (see Fig. 6 (c)). 
(a)

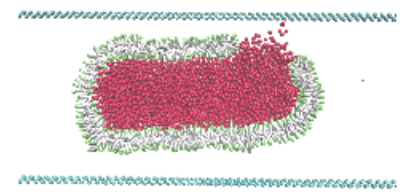

(b)

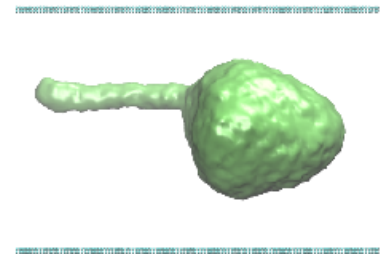

(c)

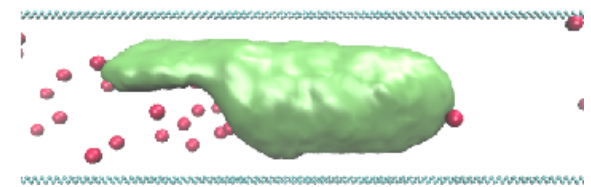

FIG. 6. Screen shots of vesicles of various confinements and reduced volumes at high pressure gradients $(\mathrm{Ca} \geq 17)$. (a) This is a cross-sectional view of a fully-inflated vesicle undergoing rupture with confinement of $\lambda=1.0$. (b) Fully-inflated vesicles at low confinement, $\lambda=0.6$, develop tethers. As the simulation progresses, the tether will continue to lengthen and eventually disconnect from the main bulk of the vesicle. (c) Some leakage of the internal fluid is observed in a vesicle with reduced volume, $\nu=0.6$, and confinement of $\lambda=1.0$. This shape is known as a slipper.

\section{B. Deformation and rupture of vesicles with reduced volume $\nu=0.6$}

We studied the deformation of vesicles with $\nu=0.6$, the reduced volume of red blood cells (RBCs). Although a RBC contains a cytoskeleton and therefore its dynamics are expected to be very different, it is interesting to see how a system with only a lipid bilayer and the same reduced volume would behave under flow and make comparisons with RBCs. We also compare our results with other simulations of vesicles. Our shapes are plotted for different confinements and capillary numbers with the area expansion mapped onto the surface (see Fig. 7). As the capillary number is increased, the deformation of the vesicles is greater as seen from the area expansion, $\alpha_{v e s}$. At $C a=20$ the vesicles are unstable as indicated by the starred shapes and eventually break where the tail connects to the main bulk. Although these vesicles eventually break, they exist long enough to permit characterization of their shape and area expansion.

The shapes with distinct tails are slipper-like which is a non-axisymmetric shape characterized by a single tail. At high capillary numbers the single tail of these slipper-like shapes become longer and, once a critical value $C a^{*}$ is reached, will disconnect from the main body of the vesicle without much interior fluid loss. There will be two separate pieces of vesicles which are a smaller piece from the tail of the vesicle and the remaining bulk of the vesicle. The main bulk of the vesicle is similar to a fully-inflated vesicle as some of the excess area has been removed. From the area expansion, the tails of the vesicles and front tip of the vesicle experience the greatest area expansions of $10 \%$ or more as compared to a quiescent 


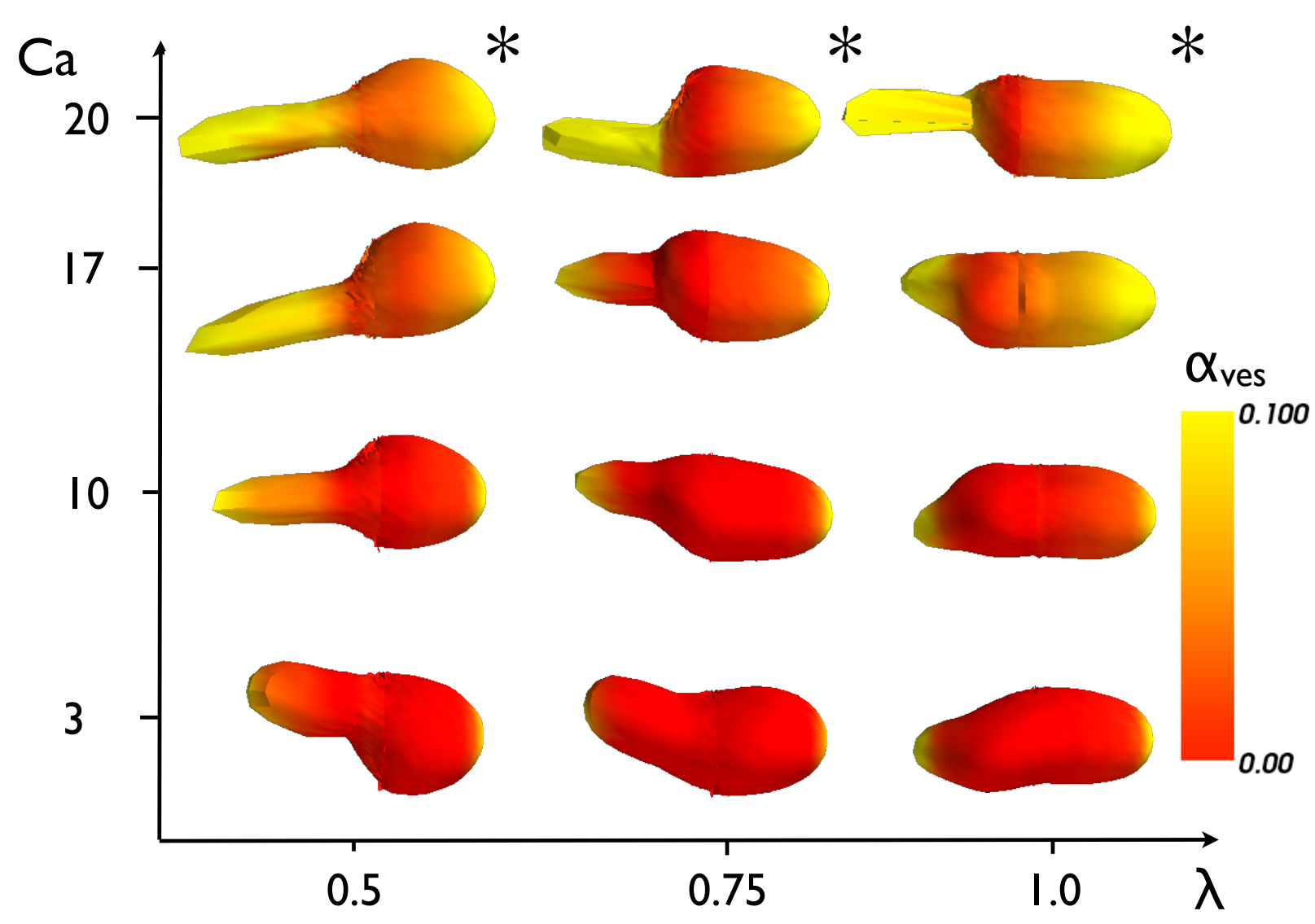

FIG. 7. Shape deformations of vesicles with $\nu=0.6$ at different flow strengths and confinements. The shapes with distinct tails are slipper-like. As the capillary number $C a$ increases, the "tails" sof these slippers elongate. The tails of the vesicles will eventually disconnect as the simulation time progresses for the $\left(^{*}\right)$ starred shapes. The area expansion, $\alpha_{v e s}$, is measured with respect to the area of a quiescent vesicle (see Eq. 2.1).

vesicle.

The area expansions as a function of confinement $\lambda$ are less differentiated as compared to the fully-inflated $\nu=1.0$ vesicles. For the fully-inflated vesicles under force with the closefitting capillary $(\lambda=1.0)$, there was a distinct pressure point at $80 \%$ of the length of the vesicle. At low capillary number (the bottom two rows of Fig. 7) the shape configurations differ between the different confinements while the area expansions are about the same amount. There is detectable stretching at the front and along the tails of the vesicles, but they are similar in all the confinements shown. The "floppiness" of the $\nu=0.6$ vesicles 
allows them to adopt configurations that minimize the stretching of the bilayer. The starred shapes in Fig. 7 are unstable. As the simulation continues the tails will eventually detach.

The modelling of vesicles in three dimensions is still an active area of research with varying results depending on the model and vesicle velocity. Kaoui et al. [10] using BIM find both slipper and parachute shapes when they model vesicles with $\nu=0.7$ for various flow strengths for both vesicles in $2 \mathrm{D}$ or $3 \mathrm{D}$. In $3 \mathrm{D}$ at a lower flow strength of $\mathrm{Ca}=40$ they find a slipper shape and at higher flow strengths they find parachute shapes. Noguchi and Gompper [7] with a fluid mesh vesicle model find prolate ellipsoidal shapes. Their elastic vesicle model (which does have a shear elasticity) shows both parachute and slipper shapes, but the parachute shapes occur at a wider range of velocities. Our vesicles stabilize into slipper-like shapes at moderate to high velocities, but not into parachute-like shapes. Experimentally, Coupier et al. [6] find that stable parachute-shaped vesicles become the dominant stable shape as reduced volume decreases from 1.0 to around 0.9 , but do not experiment with vesicles of reduced volume of 0.6 .

RBCs are considerably more complex than vesicles, but it is interesting to note that slipper-like shapes have been observed for RBCs in microvasculature [34]. Our vesicles exhibit slipper-like shapes as seen in Figure 7. RBCs remodel the cytoskeleton under stress which is something our simulated vesicles cannot replicate. As the fluid flow velocity increases, the tails of our vesicles elongate and then detach from the rest of the vesicle. Such phenomenon is not known to occur to RBCs under high fluid flow. Experimentally vesicles are known to be extremely fragile and our simulations reflect that fact.

\section{DISCUSSION}

Our paper studied vesicle rupture using a coarse-grained model of a vesicle with a fluid bilayer structure and an explicit fluid in which it is possible to break the bilayer under high stresses. We showed that the eccentricity of our fully-inflated vesicles as a function of velocity correspond to experimental vesicles and therefore our vesicles show similar rheological properties to real-world vesicles. Also our vesicles show similar rheological properties to RBCs when comparing the velocity of our vesicles to the average fluid velocity. We measured the area expansion as a function of the length of the vesicle. This showed a key location in which stress builds up in the bilayer as the velocity of the vesicle increases. We 
varied the confinement of the vesicle and were able to show that more confined vesicles are under greater stress than less confined vesicles for the same capillary numbers. In fact, less confined vesicles do not build up enough stress to rupture like more confined vesicles, but instead they form tethers and break from the back of the vesicle.

A result from this paper is the change in eccentricity of vesicles as a function of velocity. The purpose of this was to show a correspondence between the paper from Vitkova [5] as they measured the eccentricity of GUVs as a function of velocity. Our velocity was represented in the reduced (naturalized) units of our coarse-grained model. If our velocities are converted into SI units, the velocities are several magnitudes faster than they would be in typical experiments. The reason for this is that the density of our fluid is about an order of magnitude less than water. In order to keep computation times low, the number of particles in our simulation was kept low and this lowered the density of our fluid. However, our flow is laminar and the vesicles show the same shape deformations as in experiment. The velocity of our fluid needs to be larger to impart the same forces to the vesicles than would be found in real-world experiments. Also nano-sized vesicles such as ours are more stable than micron-sized vesicles therefore would require more force to bend and rupture. A third factor that accounts for the faster speeds is that in coarse-grained simulations everything just moves faster. Several degrees of freedom are lost in a coarse-grained model and even when more details are included [35] speeds are faster than in the real-world.

Another result of this paper was the local area expansion as a function of length with increasing capillary number (which is proportional to the velocity of the vesicle). This dimensionless capillary number is used in several other simulation works [8], [10], [6]. This capillary constant can be expressed as the product of the relaxation time of the vesicles and a shear rate. This relaxation time, $\tau_{b e n d}$, of the vesicle is an overestimate as it assumes the relaxation occurs on the length scale, $R_{0}$, of the vesicle [36]. Consequently the capillary number is not independent of the vesicle size $R_{0}$. Our capillary numbers range from 3 to 20 . However capillary numbers can span several decades [6] and the GUVs in the Vitkova et al. experiment had capillary numbers of around 1000 [5]. Nevertheless, our greater than one capillary numbers show the dominance of the fluid stress over the reaction of the vesicle.

The fluid flow around our vesicles is Poiseuille and laminar and should create stresses in same manner as in real-world experiments. Experimentally, nano-sized vesicles are very stable and difficult to break and therefore our large expansions are a reflection of that. The 
area expansion of our bilayer can go as high as $25 \%$ before breaking. This is a much greater expansion than micron-sized vesicles can withstand. Experimentally a $5 \%$ area expansion is the maximum a bilayer can stretch before lysis [31]. However, one should expect the stress profile of our vesicles would be applicable to larger vesicles as the shape deformations are similar and the response of our vesicles to the fluid flow is similar to real-world experiments. The purpose of our study shows how the stress builds up around the vesicle in flow.

The forces experienced by more confined vesicles is greater than the least confined vesicles for the same capillary number as seen in Figure 4. Capillary number is proportional to the velocity of the vesicle so at the same velocity a more confined vesicle would be more likely to rupture. This principle is not surprising as confined vesicles should experience more force. For less confined vesicles, there is no build-up of stress near the front of the vesicles that leads to rupture of the bilayer. Instead, there is area expansion throughout the bilayer and at the rear tethers form. A tether is a long piece of membrane extruded from the bulk of the vesicle and occurs in various experiments [37.

We modelled partially-inflated vesicles with a reduced volume of $\nu=0.6$ for various capillary numbers and confinements. Our primary shape, found for confinements of 0.5 to 1.0 and various flow rates, is a non-symmetric slipper shape. This shape has a distinct tail that is most prominent at higher flow rates. In fact, these vesicles break by having the tail elongate to the point that it breaks from the main bulk of the vesicle. In general, for vesicles with reduced volume $\nu=0.6$, their shape deformations and other dynamics are known from simulations only as most experiments use fully-inflated or close to fully-inflated vesicles. Our modelling provides another avenue for simulating vesicles in a three-dimensional context. There are three-dimensional simulation models of vesicles, but ours uses a lipid bilayer rather than assuming a mesh model. We did not find parachute-shaped vesicles as some simulations have found, but this could have been because our vesicles were too deformable and/or our confinement values were too low. The modelling of vesicles with the reduced volume of around 0.6 is still an active area of research.

\section{CONCLUSION}

Our model for vesicles is unique in that the membrane is a fluid bilayer and the interior and exterior fluid are explicit. This allows for a proper response of the membrane to imposed 
stresses making possible the study of rupture. For fully-inflated vesicles, our bullet-shaped vesicles are similar to what is found in experimental works [5, 6]. By analyzing the area expansion of our lipids, we are able to deduce the locations of peak stress which ultimately will be the point of rupture in near the front, but not at the tip. For these fully-inflated vesicles the location of peak stress is at 0.8 of the length of the vesicle measured from the back. For vesicles with the reduced volume of RBCs, $\nu=0.6$, we are also able to show the areas of the highest stress.

Vesicles are manufactured for medical, biological, and experimental purposes and are often known as liposomes in this context. Liposomes can be designed as drug-delivery vehicles in which the therapeutic agent is enclosed in the interior of the vesicle. This study of the rupture of fully-inflated vesicles in confined channels could be applicable to drugdelivery. For instance, in cases of ischemia there is a constriction in blood flow and the rupture of liposomes due to confinement could be applicable. Indeed there have already been several liposomal-enclosed medicines that have been applied in the case of ischemia of the brain (such as in the case of stroke) [38] and of the heart (as in the case of atherosclerosis) [39].

Our method of exploring vesicle dynamics in strong flow allows for a fluid bilayer which is capable of quick remodelling under stress. This is unique as most numerical studies rely on the limited area expansion of the bilayer to model shape deformations. It is possible that, if a coarse-grained, molecular dynamics simulation is accepted as a method for modelling vesicles or RBCs, it could be further expanded to incorporate more details such as more realistic lipids and/or a cytoskeleton attached to the bilayer.

It is hopeful that this study of rupture could inspire experiments to verify some of these dynamics and that these results could be applied in the design of liposomal-based medicines.

[1] V. P. Torchilin, Nat. Rev. Drug Discov., 4, 145 (2005).

[2] T. Lian and R. J Ho, J. Pharm. Sci., 90, 667 (2001).

[3] D. Lasic, Trends Biotechnol., 16, 307 (1998),

[4] D. Boal, Mechanics of the Cell, 2nd edition (Cambridge University Press, 2012).

[5] V. Vitkova, M. Mader, and T. Podgorski, Europhys. Lett., 68, 398 (2004). 
[6] G. Coupier, A. Farutin, C. Minetti, T. Podgorski, and C. Misbah, Phys. Rev. Lett., 108, $178106(2012)$.

[7] H. Noguchi and G. Gompper, Proc. Nat. Acad. Sci. U.S.A., 102, 14159 (2005).

[8] B. Kaoui, G. H. Ristow, I. Cantat, C. Misbah, and W. Zimmermann, Phys. Rev. E, 77, 021903 (2008)

[9] G. Danker, P. M. Vlahovska, and C. Misbah, Phys. Rev. Lett., 102, 148102 (2009).

[10] B. Kaoui, N. Tahiri, T. Biben, H. Ez-Zahraouy, A. Benyoussef, G. Biros, and C. Misbah, Phys. Rev. E, 84, 041906 (2011).

[11] T. Biben, A. Farutin, and C. Misbah, Phys Rev E, 83, 031921 (2011).

[12] B. Kaoui, J. Harting, and C. Misbah, Phys. Rev. E, 83, 066319 (2011).

[13] A. Farutin and C. Misbah, Phys. Rev. E, 84, 011902 (2011).

[14] A. Farutin, O. Aouane, and C. Misbah, Phys. Rev. E, 85, 061922 (2012).

[15] D. Abreu, M. Levant, V. Steinberg, and U. Seifert, Adv. Colloid Interface Sci., 208, 129 (2014).

[16] D. J. Quinn, I. Pivkin, S. Y. Wong, K.-H. Chiam, M. Dao, G. E. Karniadakis, and S. Suresh, Ann. Biomed. Eng., 39, 1041 (2011).

[17] G. Tomaiuolo, M. Simeone, V. Martinelli, B. Rotoli, and S. Guido, Soft Matter, 5, 3736 (2009).

[18] W. Helfrich, Z. Naturforsch. C., 28, 693 (1973).

[19] S. K. Veerapaneni, D. Gueyffier, D. Zorin, and G. Biros, J. Comput. Phys., 228, 2334 (2009).

[20] S. K. Veerapaneni, A. Rahimian, G. Biros, and D. Zorin, J. Comput. Phys., 230, 5610 (2011).

[21] J. L. McWhirter, H. Noguchi, and G. Gompper, Proc. Natl. Acad. Sci. U. S. A., 106, 6039 (2009).

[22] M. A. Idiart and Y. Levin, Phys. Rev. E, 69, 061922 (2004),

[23] L. Fournier and B. Joós, Phys. Rev. E, 67, 051908 (2003).

[24] P.-A. Boucher, B. Joós, M. J. Zuckermann, and L. Fournier, Biophys. J., 92, 4344 (2007),

[25] E. Evans, V. Heinrich, F. Ludwig, and W. Rawicz, Biophys. J., 85, 2342 (2003).

[26] P. Marmottant, T. Biben, and S. Hilgenfeldt, Proc. R. Soc. A, 464, 1781 (2008),

[27] M. Bertrand and B. Joós, Phys. Rev. E, 85, 051910 (2012).

[28] B. M. Barlow, M. Bertrand, and B. Joós, Phys. Rev. E, 85, 052408 (2016).

[29] R. Goetz and R. Lipowsky, J. Chem. Phys., 108, 7397 (1998). 
[30] S. J. Marrink and A. E. Mark, J. Am. Chem. Soc., 125, 15233 (2003), ISSN 0002-7863.

[31] W. Rawicz, K. C. Olbrich, T. McIntosh, D. Needham, and E. Evans, Biophys. J., 79, 328 $(2000)$.

[32] N. Amenta, M. Bern, and M. Kamvysselis, Proceedings of the 25th Annual Conference on Computer Graphics and Interactive Techniques, 415 (1998).

[33] K. Olbrich, W. Rawicz, D. Needham, and E. Evans, Biophys. J., 79, 321 (2000), ISSN 00063495 .

[34] R. Skalak and P. I. Branemark, Science, 164, 717 (1969).

[35] S. J. Marrink, H. J. Risselada, S. Yefimov, D. P. Tieleman, and A. H. de Vries, J. Phys. Chem. B, 111, 7812 (2007).

[36] H. Zhou, B. B. Gabilondo, W. Losert, and W. van de Water, Phys. Rev. E, 83, 011905 (2011).

[37] B. Bozic, S. Svetina, B. Zeks, and R. Waugh, Biophys. J., 61, 963 (1992).

[38] M. Yokota, E. Tani, S. Tsubuki, I. Yamaura, I. Nakagaki, S. Hori, and T. C. Saido, Brain Res., 819, 8 (1999).

[39] D. D. Verma, T. S. Levchenko, E. A. Bernstein, and V. P. Torchilin, J. Control Release, 108, $460(2005)$. 